International Journal of Pure and Applied Mathematics

Volume 107 No. 3 2016, 601-614

ISSN: 1311-8080 (printed version); ISSN: 1314-3395 (on-line version)

url: http://www.ijpam.eu

doi: $10.12732 /$ ijpam.v107i3.9

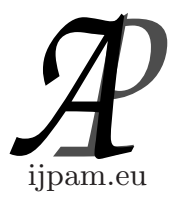

\title{
FUZZY VERTEX ORDER COLOURING
}

\author{
A. Nagoor Gani ${ }^{1 \S}$, B. Fathima Kani ${ }^{2}$ \\ ${ }^{1,2}$ P.G. and Research Department of Mathematics \\ Jamal Mohamed College (Autonomous) \\ Tirchirappalli, 620 020, Tamilnadu, INDIA
}

\begin{abstract}
In this paper three types of strong vertices are introduced.A necessary and sufficient conditions for a vertex to be strong have been given. Some of the properties of $\alpha$ strong, $\beta$-strong and $\gamma$-strong vertices are discussed.Also the concept of vertex order colouring is introduced.An algorithm for vertex order colouring is also given at the end of the chapter.
\end{abstract}

AMS Subject Classification: $03 \mathrm{E} 72,05 \mathrm{C} 72$

Key Words: $\alpha$-strong vertex, $\beta$-strong vertex, $\gamma$-strong vertex, underlying crisp graph, complete fuzzy graph, regular graph, chromatic number

\section{Introduction}

A graph is convenient way of representing information involving relationship between objects. Each object is represented by a vertex and the relationship between them is represented by an edge. Relationship among the objects need not always be precisely defined. So when we think of an imprecise concept, the fuzziness arises.

Fuzzy graphs were introduced by Rosenfeld in [12], ten years after Zadeh's in [16] land mark paper Fuzzy Sets. Fuzzy graph theory is now finding numerous applications in modern science and technology es specially in the fields of information theory, neural networks, expert systems, cluster analysis, medical diagnosis, control theory etc. Rosenfeld has obtained the fuzzy analogues of

Received: January 10, 2016

Published: April 21, 2016

$\S$ Correspondence author (c) 2016 Academic Publications, Ltd.

url: www.acadpubl.eu 
several basic graph theoretic concepts like bridges, paths, cycles, trees and connectedness established some of their properties. Bhattacharya has established some connectivity concepts regarding fuzzy cut nodes and fuzzy bridges.

Graph colouring is one of the most important concepts in graph theory and is used in many real time applications like job scheduling, aircraft scheduling, computer network security, map colouring and G. S. M mobile phone networks, automatic channel allocation for small wireless local area networks.

A colouring of a crisp graph $\mathrm{G}$ : $(\mathrm{V}, \mathrm{E})$ is an assignment of colours to its vertices so that no two adjacent vertices have the same colour. The set of all vertices with any one colour is independent and is called colour class. A k colouring of a graph $\mathrm{G}$ uses $\mathrm{k}$ colours and thereby partitions $\mathrm{V}$ into $\mathrm{k}$ colour classes. The chromatic number is defined as the minimum number $\mathrm{k}$ for which $\mathrm{G}$ has a proper $\mathrm{k}$ colouring. . In many real world problems information about the problem is not clear. Therefore we have to design fuzzy graph model.

The concept of chromatic number of fuzzy graphs was introduced by Munoz et al. Later Eslahchi and Onagh in [3] defined fuzzy colouring of fuzzy graphs and defined fuzzy chromatic number. Fuzzy graph colouring is one of the most important problems of fuzzy graph theory and is used in many real life applications like traffic light control, exam scheduling, register allocation. Fuzzy colouring is an assignment of colours to vertices in which no two strong adjacent vertices have the same colour. The fuzzy chromatic number of a fuzzy graph $\mathrm{G}$ is the minimum number $\mathrm{k}$ for which $\mathrm{G}$ has $\mathrm{k}$ colours to vertices where no two strong adjacent vertices have the same colour.

In this paper basic definitions related to fuzzy graphs and fuzzy graph colouring is given in the second chapter. Three types of strong vertices $\{\alpha-$ strong, $\beta$ - strong, $\gamma$ - strong $\}$ and vertex order colouring are defined in the third chapter. Some of the properties of strong vertices are given in the fourth chapter. In the fifth chapter an algorithm for vertex order colouring with example is given. Bibliography is given at the end of the conclusion.

\section{Basic Definitions}

Let $V$ be a finite non empty set and $E$ be the collection of two element subsets of $V$. A fuzzy graph $G=(\sigma, \mu)$ is a set with two functions $\sigma: V \rightarrow[0,1]$ and $\mu: E \rightarrow[0,1]$ such that $\mu(u, v) \leq \sigma(u) \wedge \sigma(v)$ for all $u, v \in V$. The underlying crisp graph of $\mathrm{G}$ by $G *:(\sigma *, \mu *)$, where $\sigma *=\{u \in V: \sigma(u)>0\}$ and $\mu *=\{(u, v) \in V * V: \mu(u, v)>0\}$ The scalar cardinality of $S \subseteq V$ is defined by $\sum_{u \in S} \sigma(u)$. The order (denoted by p) and size (denoted by q) of a fuzzy 
graph $G$ are the scalar cardinality of $\sigma$ and $\mu$ respectively. An edge $e=(u, v)$ of a fuzzy graph is called an effective edge if $\mu(u, v)=\sigma(u) \wedge \sigma(v)$. If $e=(u, v)$ is an effective edge, then $u$ and $v$ are adjacent vertices and $e$ is incident with $u$ and $v$.

A fuzzy graph $G=(\sigma, \mu)$ is said to be $M$-strong fuzzy graph if $\mu(u, v)=$ $\sigma(u) \wedge \sigma(v)$ for all $(u, v) \in E$. That is, In an M-strong fuzzy graph every edge is an effective edge. A fuzzy graph $G=(\sigma, \mu)$ is said to be complete fuzzy graph if $\mu(u, v)=\sigma(u) \wedge \sigma(v)$ for all $u, v \in V$. That is, In a complete fuzzy graph every pair of vertices should have an effective edge. Let $u, v \in V$ and $e=(u, v) \in E$ then $N(u)=\{v \in V: \mu(u, v)=\sigma(u) \wedge \sigma(v)\}$ is called open neighbourhood of $u$ and $N[u]=N(u) \cup\{u\}$ is called closed neighbourhood of $u . N[e]=N(u) \cup N(v)$ is called closed neighbourhood of $e$. If $N(u)=\phi$ then $\mathrm{u}$ is said to be isolated vertex.

The neighbourhood degree of a vertex $u$ is defined to be the sum of the weights of the vertices adjacent to $u$ and is denoted by $d_{N}(u)$, the minimum neighbourhood degree is $\delta_{N}(u)=\min \left\{d_{N}(u): u \in V\right\}$ and the maximum neighbourhood degree is $\Delta_{N}(G)=\max \left\{d_{N}(u): u \in V\right\}$.

A path in a fuzzy graph $G$ is a sequence of distinct vertices $u_{0}, u_{1}, u_{2}, \ldots, u_{n}$ such that $\mu\left(u_{i-1}, u_{i}\right)=\sigma\left(u_{i-1}\right) \wedge \sigma\left(u_{i}\right), 1 \leq i \leq n, n>0$ is called the length of the path. The path in a fuzzy graph is called a fuzzy cycle if $u_{0}=u_{n}, n \geq 3$. A fuzzy graph is said to be cyclic if it contains at least one cycle, otherwise it is called acyclic.

A fuzzy graph is said to be connected if there exists at least one path between every pair of vertices. A connected acyclic fuzzy graph is said to be a fuzzy tree.

A vertex in a fuzzy graph having only one neighbour is called a pendent vertex. An edge in a fuzzy graph incident with a pendent vertex is called a pendent edge. Otherwise it is called non-pendent edge. A vertex in a fuzzy graph adjacent to the pendent vertices is called a support of the pendent edges.

The strength of a path $P$ is defined as $\wedge_{i=1}^{n} \mu\left(v_{i}, v_{i+1}\right)$

\section{Vertex Order Colouring}

Definition 3.1. Adjacent vertices to the vertex $\mathrm{v}$ is $\mathrm{A}(\mathrm{v})=\left\{u \in V / \frac{1}{2}[\sigma(u) \wedge\right.$ $\sigma(v)] \leq \mu(u v)\} A_{s}(v)=\{u \in A(v) \mid d(v) \geq d(u)\} A_{w}(v)=\{u \in A(v) \mid d(v)<d(u)\}$.

Definition 3.2. Strong degree of the vertex v is $d_{s}(v)=\sum_{u \in A_{s}(v)} \mu(u v)$ Weak degree of the vertex $\mathrm{v}$ is $d_{w}(v)=\sum_{u \in A_{w}(v)} \mu(u v)$. 
Definition 3.3. A vertex $\mathrm{v}$ is called strong silent if $d_{s}(v)=0$ and weak silent if $d_{w}(v)=0$.

Definition 3.4. A vertex $\mathrm{v} \in \mathrm{V}$ of a fuzzy graph $G:(\sigma, \mu)$ is said to be $\alpha$-strong, if for every $\mathrm{v} \in \mathrm{A}(\mathrm{V})$ satisfies $\mathrm{d}(\mathrm{v}) \geq \mathrm{d}(\mathrm{A}(\mathrm{v}))$.

Definition 3.5. A vertex $\mathrm{v} \in \mathrm{V}$ of a fuzzy graph $G:(\sigma, \mu)$ is said to be $\gamma$-strong or weak vertex, if for every $\mathrm{v} \in \mathrm{A}(\mathrm{V})$ satisfies $\mathrm{d}(\mathrm{v})<\mathrm{d}(\mathrm{A}(\mathrm{v}))$.

Definition 3.6. A vertex which satisfy the condition of $\alpha$-strong and $\gamma$-strong is said to be $\beta$-strong vertex.

Note 1. All Alpha strong vertices are weak silent and vice versa.

Note 2. All Gamma strong vertices are strong silent and vice versa.

Definition 3.7. Collection of $\alpha$-strong vertices are denoted by $S_{\alpha}(V)$, where $S_{\alpha}(V)=\{u \in V / d(v) \geq d(A(v))\}$.

Definition 3.8. Collection of $\beta$-strong vertices are denoted by $\beta(V)$, where $\beta(V)=\{u \in V / d(v)<d(A(v))$ and $d(v) \geq d(A(v))\}$.

Definition 3.9. Collection of $\gamma$-strong vertices are denoted by $W_{\gamma}(V)$, where $W_{\gamma}(V)=\{u \in V / d(v)<d(A(v))\}$.

Definition 3.10. Fuzzy graph parameters are:

1. Maximum $\alpha$-strong vertex of a fuzzy graph $\Delta_{S_{\alpha}}(G)$.

Minimum $\alpha$-strong vertex of a fuzzy graph $\delta_{S_{\alpha}}(G)$.

2. Maximum $\beta$-strong vertex of a fuzzy graph $\Delta_{\beta}(G)$.

Minimum $\beta$-strong vertex of a fuzzy graph $\delta_{\beta}(G)$.

3. Maximum $\gamma$-strong vertex of a fuzzy graph $\Delta_{W_{\gamma}}(G)$.

Minimum $\gamma$-strong vertex of a fuzzy graph $\delta_{W_{\gamma}}(G)$.

Definition 3.11. 1. A vertex $\mathrm{v}$ is strictly $\alpha$ - strong if $d(v)>d(u)$, $\forall \mathrm{u} \in \mathrm{A}(\mathrm{v})$.

2. A $\gamma$-strong vertex is always strict. 
3. A $\beta$-strong vertex is both strong and weak.

Definition 3.12. The effective degree of a vertex $v$ is defined to be the sum of the membership values of the effective edges incident with $\mathrm{v}$ and is denoted by $d_{E}(V)$.

Definition 3.13. 1. The minimum effective degree of $\mathrm{G}$ is $\delta_{E}(G)$, it is defined by $\delta_{E}(G)=\wedge\left\{d_{E}(V) / v \in V\right\}$.

2. The maximum effective degree of $\mathrm{G}$ is $\Delta_{E}(G)$, it is defined by $\Delta_{E}(G)=$ $\vee\left\{d_{E}(V) / v \in V\right\}$.

Note 3. Maximum effective degree of a fuzzy graph $\mathrm{G}$ is $\alpha$ - strong vertex.

Note 4. Minimum effective degree of a fuzzy graph $\mathrm{G}$ is $\gamma$ - strong vertex.

Definition 3.14. Later Eslahchi and Onagh introduced fuzzy colouring of fuzzy graphs as follows. A family $\Gamma=\left\{\gamma_{1}, \gamma_{2}, \ldots, \gamma_{k}\right\}$ of fuzzy sets on a set $\mathrm{V}$ is called a k-fuzzy colouring of $\mathrm{G}=(V, \sigma, \mu)$ if:

(i) $\vee \Gamma=\sigma$,

(ii) $\gamma_{i} \wedge \gamma_{j}=0$,

(iii)For every strong edge (x, y)(i. e $\mu(x, y)>0)$ of $\mathrm{G}, \min \left\{\gamma_{i}(x), \gamma_{i}(y)\right\}=0$, $(1 \leq \mathrm{i} \leq \mathrm{k})$.

The minimum number $\mathrm{k}$ for which there exists a k-fuzzy colouring is called the fuzzy chromatic number of $\mathrm{G}$, denoted as $\chi^{f}(G)$.

Definition 3.15. A fuzzy vertex order colouring is assigning the increasing sequence of $\mathrm{k}$-colours to all the vertices by using the strong degree of vertices. Increasing sequence of colours belongs to the order $S_{\alpha}(V)<\beta(V)<W_{\gamma}(V)$.

Definition 3.16. A family $\xi=\left\{S_{\alpha}(V), \beta(V), W_{\gamma}(V)\right\}$ of strong vertices on a set $\mathrm{V}$ is called a fuzzy vertex order colouring of $\mathrm{G}=(V, \sigma, \mu)$ if:

(i) $\bigcup \xi=\sigma$,

(ii) $\bigcap \xi=\phi$,

(iii)For every $(\mu(x, y)>0)$ of $\mathrm{G}$, if $\mathrm{x}, \mathrm{y} \in S_{\alpha}(V)$ (or) $\mathrm{x}, \mathrm{y} \in \beta(V)$, then $\mathrm{c}(\mathrm{x}) \neq \mathrm{c}(\mathrm{y})$, where $\mathrm{c}(\mathrm{x})$ is the colour of $\mathrm{x}$, 
(iv)For every $(\mu(x, y)>0)$ of $\mathrm{G}$, if $\mathrm{x}, \mathrm{y} \in w_{\gamma}(V)$, then $\mathrm{c}(\mathrm{x})=\mathrm{c}(\mathrm{y})$ and make $(\mu(x, y)=0)$,

$(\mathrm{v})$ For every $(\mu(x, y)=0)$ of $\mathrm{G}$, if $\mathrm{x}, \mathrm{y} \in \xi$, then $\mathrm{c}(\mathrm{x})=\mathrm{c}(\mathrm{y})$.

The minimum number $\mathrm{k}$ for which there exists a $\mathrm{k}$-fuzzy vertex order colouring is called the fuzzy chromatic number of $\mathrm{G}$, denoted as $\chi^{f_{o}}(G)$.

Example 1. Consider the fuzzy graph $\mathrm{G}=(V, \sigma, \mu)$ with 7 vertices ( $\mathrm{u}, \mathrm{v}$, $\mathrm{w}, \mathrm{x}, \mathrm{y}, \mathrm{z}, \mathrm{p})$ and 10 edges $\mu(u v), \mu(u z), \mu(z p), \mu(v p), \mu(u p), \mu(z y), \mu(y x)$, $\mu(p x), \mu(w y), \mu(v w)$.

Membership values of $\sigma$ and $\mu$ are

$$
\begin{gathered}
\sigma(u)=0.4, \quad \sigma(v)=1, \quad \sigma(w)=0.8, \quad \sigma(x)=0.3 \\
\sigma(y)=0.7, \quad \sigma(z)=1, \quad \sigma(p)=1, \quad \mu(u v)=0.1, \quad \mu(u z)=0.3, \\
\mu(z p)=0.9, \quad \mu(v p)=0.5, \quad \mu(u p)=0.3, \quad \mu(z y)=0.3 \\
\mu(y x)=0.2, \quad \mu(p x)=0.3, \quad \mu(w y)=0.6, \quad \mu(v w)=0.8 .
\end{gathered}
$$

$\mathrm{u}$ is not Adjacent to $\mathrm{v}$ and $\mathrm{z}$ not adjacent to $\mathrm{y}$ since $\left\{\frac{1}{2}[\sigma(u) \wedge \sigma(v)]>\mu(u v)\right\}$ and $\left\{\frac{1}{2}[\sigma(z) \wedge \sigma(y)]>\mu(z y)\right\}$. Hence remove the edges $\mu(u v), \mu(z y)$ from the fuzzy graph.

From the definitions of $\alpha$-strong, $\beta$-strong and $\gamma$-strong vertices, $S_{\alpha}(V)=\{\mathrm{w}$, p) $\beta(V)=\{\mathrm{y}, \mathrm{z}\} W_{\gamma}(V)=\{\mathrm{u}, \mathrm{v}, \mathrm{x}\}$. Thus vertex order colouring of the fuzzy graph is:

$\mathrm{w}-1, \mathrm{p}-1$, Since there is no edge between $\mathrm{w}$ and $\mathrm{p}$.

$\mathrm{y}-2, \mathrm{z}-2$, Since there is no edge between $\mathrm{y}$ and $\mathrm{z}$.

$\mathrm{u}-3, \mathrm{v}-3, \mathrm{x}-3$, Since there is no edge between $\mathrm{u}, \mathrm{v}$ and $\mathrm{x}$.

The chromatic number of the fuzzy graph is $\chi^{f_{o}}(G)=3$.

\section{Properties of $\alpha$-Strong, $\beta$-Strong, $\gamma$-Strong Vertices}

Theorem 4.1. Let $G:(V, \sigma, \mu)$ be a fuzzy graph. Then for any vertex $v \in V, d(v)=d_{s}(v)+d_{w}(v)$. 
Proof. For any vertex $\mathrm{v} \in \mathrm{V}, \mathrm{A}(\mathrm{v})$ is adjacent vertices for the vertex v. $A_{s}(v)$ is strong adjacent vertices to the vertex $\mathrm{v}, A_{w}(v)$ is weak adjacent vertices to the vertex $\mathrm{v}$.

Let $\mathrm{A}=\mathrm{A}(\mathrm{v}), \mathrm{S}=\mathrm{A}_{S}(v), \mathrm{W}=A_{w}(v)$.

We observe that $\mathrm{A}=\mathrm{S} \cup \mathrm{W}$ and

$|A|=|S \cup W| d(v)=|A|=|S|+|W|+|S \cap W|=d_{s}(v)+d_{w}(v)-\phi$.

Hence $\mathrm{d}(\mathrm{v})=d_{s}(v)+d_{w}(v)$.

Theorem 4.2. For any fuzzy graph $G:(V, \sigma, \mu), \sum_{v \in V}\left[d_{s}(v)+d_{w}(v)\right]$ is an even number.

Proof. For any vertex v, $\mathrm{d}(\mathrm{v})=d_{s}(v)+d_{w}(v)$. Hence

$$
\sum_{v \in V} d(v)=\sum_{v \in V}\left[d_{s}(v)+d_{w}(v)\right] .
$$

It is known that

$$
\sum_{v \in V} d(v)=2 \sum_{v \in V, u \neq v} \mu(u v) .
$$

This implies that $\sum_{v \in V} d_{s}(v)+d_{w}(v)$ is an even number.

Theorem 4.3. Let $G:(V, \sigma, \mu)$ be a fuzzy graph then:

(i) $A$ vertex $v \in V$ is $\alpha$-strong iff $d(v)=d_{s}(v)$,

(ii) A vertex $v \in V$ is $\gamma$-strong iff $d(v)=d_{w}(v)$,

(iii) $A$ vertex $v \in V$ is $\beta$-strong iff $d(v) \geq d(u)$ for some $u \in A(v)$ and $d(v)<d(w)$, for some $w \in A(v)$,

(iv) $A$ vertex $v \in V$ is strictly $\alpha$-strong iff $d_{w}(v)=0$,

$(\mathbf{v}) A$ vertex $v \in V$ is strictly $\gamma$-strong iff $d_{s}(v)=0$.

Proof. (i) Given G:(V, $\sigma, \mu)$ be a fuzzy graph.

Let $\mathrm{v} \in \mathrm{V}$ be a $\alpha$-strong vertex. Then $\mathrm{d}(\mathrm{v}) \geq \mathrm{d}(\mathrm{u})$ for every $\mathrm{u} \in \mathrm{A}(\mathrm{v})$. Hence $\mathrm{A}_{w}(\mathrm{v})=\phi$. Therefore $d_{w}(v)=0, \forall \mathrm{v} \in A_{w}(\mathrm{v})$.

We know that $\mathrm{d}(\mathrm{v})=d_{s}(v)+d_{w}(v)$.

$$
\begin{gathered}
\Rightarrow d(v)=d_{s}(v), \quad \forall v \in A(v) . \\
\Rightarrow d(v)=d_{s}(v) .
\end{gathered}
$$


Converse follows from the Note 1.

(ii) Let $\mathrm{v} \in \mathrm{V}$ be a $\gamma$-strong vertex. Then

$$
d(v)<d(u), \quad \forall u \in A(v)
$$

Hence $A_{s}(v)=\phi$. Therefore $d_{s}(v)=0$. This implies that $\mathrm{d}(\mathrm{v})=d_{w}(v), \forall \mathrm{v} \in \mathrm{A}(\mathrm{v})$. Converse follows from the Note 1 .

(iii) Let $\mathrm{v}$ be a $\beta$-strong vertex. To prove $\mathrm{d}(\mathrm{v}) \geq \mathrm{d}(\mathrm{u})$, for some $\mathrm{u} \in \mathrm{A}(\mathrm{v})$ and $d(v)<d(w)$, for some $\mathrm{w} \in \mathrm{A}(\mathrm{v})$. Suppose $\mathrm{d}(\mathrm{v}) \geq \mathrm{d}(\mathrm{u})$, for some $\mathrm{u} \in \mathrm{A}(\mathrm{v})$ does not hold. Then $d(v)<d(w)$, for every $\mathrm{w} \in \mathrm{A}(\mathrm{v})$ hold. Hence $\mathrm{v}$ is $\gamma$-strong vertex, which gives a contradiction.

Suppose if $d(v)<d(w)$, for some $\mathrm{w} \in \mathrm{A}(\mathrm{v})$ does not hold. Then $\mathrm{d}(\mathrm{v}) \geq \mathrm{d}(\mathrm{w})$, for every $\mathrm{w} \in \mathrm{A}(\mathrm{v})$ hold, implies $\mathrm{v}$ is the $\alpha$-strong vertex, which gives a contradiction. This implies that, if $\mathrm{v}$ is $\beta$-strong vertex, then $\mathrm{d}(\mathrm{v}) \geq \mathrm{d}(\mathrm{u})$, for some $\mathrm{u} \in \mathrm{A}(\mathrm{v})$ and $d(v)<d(w)$, for some $\mathrm{w} \in \mathrm{A}(\mathrm{v})$ holds.

Converse follows from the Definition 3.6.

The proof of (iv) and (v) are also follows from the Note 1. Hence the theorem proved.

Theorem 4.4. Let $G$ be a fuzzy graph. If $G$ is regular fuzzy graph then all vertices of $G$ is $\alpha$-strong vertices.

Proof. Given $\mathrm{G}$ be a regular fuzzy graph with vertices $\mathrm{V}$ and edges $\mathrm{E}$, whose membership values are denoted by $(\sigma *, \mu *)$.

Clearly $\mathrm{d}(\mathrm{v})=\mathrm{c}, \forall \mathrm{v} \in \mathrm{V}$, where $\mathrm{c}$ is a constant real value. Hence comparing the degree of all vertices to its adjacent vertices, the degrees are equal. Hence if fuzzy graph $\mathrm{G}$ have n-number of vertices, then $\alpha$-strong vertices of the fuzzy graph $\mathrm{G}$ is $\mathrm{n}$.

Theorem 4.5. Let $G$ be a fuzzy graph, whose underlying crisp graph $G^{*}$ is regular. The maximum $\alpha$-strong vertices for the fuzzy graph is one.

Proof. Given underlying crisp graph $\mathrm{G}^{*}$ is regular. Consider the regular graph of $\mathrm{G}^{*}$ with more than two vertices.

Let $\mathrm{G}$ be the fuzzy graph with three vertices, whose underlying crisp graph $\mathrm{G}^{*}$ is regular of degree two. Assume the membership values of edges are distinct. Choose a vertex with minimum degree say $u_{1}$ and call it as $\gamma$-strong vertex.

That is

$$
d_{G}\left(u_{1}\right)<d_{G}\left(u_{2}\right)
$$


and

$$
\begin{gathered}
d_{G}\left(u_{1}\right)<d_{G}\left(u_{3}\right), \\
\mu_{12}\left(u_{1}, u_{2}\right)+\mu_{13}\left(u_{1}, u_{3}\right)<\mu_{21}\left(u_{2}, u_{1}\right)+\mu_{23}\left(u_{2}, u_{3}\right), \\
\Rightarrow \mu_{13}\left(u_{1}, u_{3}\right)<\mu_{23}\left(u_{2}, u_{3}\right) .
\end{gathered}
$$

If $\mu_{12}\left(u_{1}, u_{2}\right)$ is greater than $\mu_{13}\left(u_{1}, u_{3}\right)$, then $d_{G}\left(u_{2}\right)$ is maximum when compared with $d_{G}\left(u_{3}\right)$.

Hence the vertex $u_{2}$ is $\alpha$-strong vertex and the node $u_{3}$ is $\beta$-strong node. Unless the node $u_{3}$ is $\alpha$-strong vertex and the node $u_{2}$ is $\beta$-strong node. Hence it is true for all fuzzy graphs, whose underlying crisp graphs are regular with even degree. Consider the fuzzy graph with odd degree, whose underlying crisp graph is regular. We know that a fuzzy graph with odd degree vertices are even say $\mathrm{m}$.

Case (i) Underlying crisp graph is complete graph.

Choose a vertex with minimum degree, let it be $u_{1}$, which is $\gamma$-strong vertex. Delete the vertex from the fuzzy graph $\mathrm{G}$. Hence we have m-1 vertices of even degree. We know that a fuzzy graph with vertices of even degree have at most one $\alpha$-strong vertex. Hence we conclude that a fuzzy sub-graph of $\mathrm{G}$, of $\mathrm{m}-1$ vertices with even degree have at most one $\alpha$-strong vertex. Thus a fuzzy graph $\mathrm{G}$ with odd degree, whose underlying crisp graph is complete have at most one $\alpha$-strong vertex.

Case (ii) Underlying crisp graph is not complete but regular graph. A fuzzy graph with odd degree vertices are even, say $\mathrm{m}$. Choose a vertex with minimum membership degree, say, let it be $u_{1}$, which is $\gamma$-strong vertex. Remove the $\gamma$ strong vertex from the fuzzy graph. After deletion the vertices with odd degree and even degree are present in the fuzzy graph G. If odd degree vertices are more than the even degree vertices then any one of odd degree vertices gives $\alpha$-strong vertex. If $\alpha$-strong vertex belong to odd degree vertices of underlying crisp graph, then delete the particular $\alpha$-strong vertex. Hence obtain the fuzzy graph with even degree.

Thus the remaining vertices have minimum degree when compared to the degree of $\alpha$-strong vertex and maximum when compared to the degree of $\gamma$ strong vertex.

It is clear that $\mathrm{m}-2$ vertices are $\beta$-strong vertex. Hence a fuzzy graph $\mathrm{G}$ whose crisp graph $\mathrm{G}^{*}$ is regular, have at most one $\alpha$-strong vertex.

Theorem 4.6. A complete fuzzy graph $G$ must have at least one pair of $\alpha$-strong vertices whose degrees are same. 
Proof. Let $G:(V, \sigma, \mu)$ be a complete fuzzy graph, where $\mathrm{V}$ is finite set, $\sigma$ is the fuzzy subset of $\mathrm{V}$ and $\mu$ is the fuzzy subset of $\mathrm{V} \times \mathrm{V}$.

Case (i) Suppose $\sigma\left(v_{i}\right)$ are equal for all $v_{i} \in V$. Obviously $\mu\left(v_{i}, v_{j}\right)$ are all equal. Hence the degree of all vertices are equal. Therefore a complete fuzzy graph must have at least one pair of $\alpha$-strong vertices whose degrees are same.

Case (ii) Suppose $\sigma\left(v_{i}\right)$ are distinct, for all $v_{i} \in V$. Since $v_{j}$ are adjacent to $v_{i}(i \neq j)$, we get the maximum degree of one pair of vertices are equal. Hence a complete fuzzy graph must have at least one pair of $\alpha$-strong vertices, whose degrees are same.

Theorem 4.7. A node $u$ is $\gamma$-strong node if the fuzzy graph $G:(\sigma, \mu)$ have at least one unique weakest arc.

Proof. Let $G:(\sigma, \mu)$ be any fuzzy graph. Let $(\mathrm{u}, \mathrm{v})$ be weakest arc. Then by definition $\mu(u, v)<\mu^{\prime} \infty(u, v)$. That is there exist at least one path $\mathrm{P}$ joining $\mathrm{u}$ and $\mathrm{v}$ and not containing the $\operatorname{arc}(\mathrm{u}, \mathrm{v})$ such that the strength of $\mathrm{P}$ greater than $\mu(u, v)$. This path together with the arc forms a cycle in which $(\mathrm{u}, \mathrm{v})$ is the unique weakest arc. Hence at least either $u(o r) v$ is $\gamma$-strong node.

Theorem 4.8. Let $G$ be a fuzzy graph, whose underlying crisp graph $G^{*}$ is regular. The following inequality holds, $\forall v \in V,\left[d_{\gamma}(v)<\operatorname{mind}_{\alpha}\left(v_{i}\right)\right]$, where $i \in Z^{+}$.

Proof. Let $G:(\sigma, \mu)$ be any fuzzy graph, whose underlying crisp graph is regular. $\mathrm{V}$ is non-empty, and $\sigma$-fuzzy subset of $\mathrm{V}$ and $\mu$-fuzzy subset of $V \times V$. Since $d_{\alpha}(v)$ is degree of $\alpha$-strong vertex, and $d_{\gamma}(v)$ is degree of $\gamma$-strong vertex. We know that minimum effective degree is less than maximum effective degree. Hence $\forall v \in V,\left[d_{\gamma}(v)<\operatorname{mind}_{\alpha}\left(v_{i}\right)\right]$, where $i \in Z^{+}$.

Theorem 4.9. The chromatic number of a fuzzy complete graph is $n$, where $n$ is the number of vertices of $G$.

Proof. Let G be a complete fuzzy graph $G:(\sigma, \mu)$. Since $\mu(u, v) \leq \sigma(u) \wedge$ $\sigma(v), \forall u, v \in V$, every pair of vertices are strongly adjacent. Degree of each vertex is $\mathrm{n}-1$. All vertices are strongly adjacent. Hence each vertex have distinct colours. Thus a complete fuzzy graph of $\mathrm{n}$ vertices have chromatic number n. 


\section{An Algorithm for Fuzzy Vertex Order Colouring}

Given a input simple fuzzy graph $\mathrm{G}$ with $\mathrm{n}$ vertices, search for a m-colouring of the vertices of the fuzzy graph.

Let $\left\{u_{1}, u_{2}, \ldots, u_{n}\right\}$ denote the fuzzy vertices of $G:(V, \sigma, \mu)$ and let $\{1,2, \ldots$, $m\} \in Z^{+}$denote the colouring values of vertices.

Step 1: Find the adjacent vertices for $u_{i}, 1 \leq i \leq n$.

Step 2: If $u_{j} \in V$ does not belongs to $A\left(u_{i}\right), j \neq i$, even when $\mu\left(u_{i} u_{j}\right)>0$, then delete an edge $\mu\left(u_{i} u_{j}\right), \forall u_{i} u_{j} \in E$.

Step 3: Find $d\left(u_{i}\right), \mathrm{i}=1,2, \ldots, \mathrm{n}$.

Step 4: Compare degree of each vertex to their degree of its adjacent vertices.

Step 5: If $d\left(u_{i}\right) \geq d\left(A\left(u_{i}\right)\right), 1 \leq i \leq n$, then put $u_{i} \in S_{\alpha}(V)$.

Else if $d\left(u_{i}\right)<d\left(A\left(u_{i}\right)\right), 1 \leq i \leq n$, then put $u_{i} \in W_{\gamma}(V)$. Else put $u_{i} \in \beta(V)$.

Step 6: Assign colours 1 to $\mathrm{j}$ from the set $Z^{+}$to $S_{\alpha}(V)$.

Step 7: If $\alpha$-strong vertices are not adjacent, then assign, same colour to all the $\alpha$-strong vertices. Otherwise assign different colours to the vertices which belongs to $S_{\alpha}(V)$.

Step 8: Assign colour values $\mathrm{j}+1$ to $\mathrm{k}$ to $\beta(\mathrm{V})$ and follows step 7 for $\beta$ strong vertices.

Step 9: Assign same value from $[\mathrm{k}+1, \mathrm{~m}]$ to all $W_{\gamma}(V)$ vertices.

Step 10: If $\gamma$-strong vertices are adjacent, then delete an edge between $\gamma$ strong vertices. Otherwise assign same colours to all the vertices which belongs to $W_{\gamma}(V)$.

Step 11: End of process.

Example. Consider the fuzzy graph. Find the adjacent vertices for all vertices. 


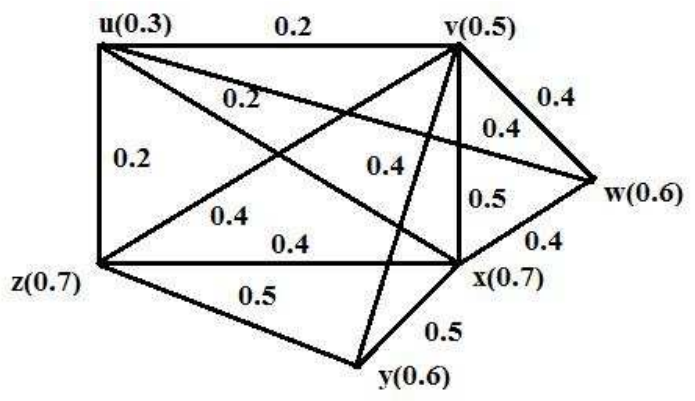

Figure 1

\begin{tabular}{|c|c|}
\hline Vertex & Adjacent Vertices \\
\hline $\mathrm{u}$ & $\mathrm{v}, \mathrm{w}, \mathrm{x}, \mathrm{z}$ \\
\hline $\mathrm{v}$ & $\mathrm{u}, \mathrm{w}, \mathrm{x} \cdot \mathrm{y} \cdot \mathrm{z}$ \\
\hline $\mathrm{w}$ & $\mathrm{u}, \mathrm{v}, \mathrm{x}$ \\
\hline $\mathrm{x}$ & $\mathrm{u}, \mathrm{v}, \mathrm{w}, \mathrm{y}, \mathrm{z}$ \\
\hline $\mathrm{y}$ & $\mathrm{v}, \mathrm{x}, \mathrm{z}$ \\
\hline $\mathrm{z}$ & $\mathrm{u}, \mathrm{v}, \mathrm{x}, \mathrm{y}$ \\
\hline
\end{tabular}

Then from the definitions of $\alpha$-strong, $\beta$-strong and $\gamma$-strong, grouping the vertices.

\begin{tabular}{|c|c|c|c|c|}
\hline Vertex & Degree & $\begin{array}{c}\text { Alpha Strong } \\
\text { Vertices }\end{array}$ & $\begin{array}{c}\text { Beta Strong } \\
\text { Vertices }\end{array}$ & $\begin{array}{c}\text { Gamma Strong } \\
\text { Vertices }\end{array}$ \\
\hline $\mathrm{u}$ & 1.0 & - & - & $\mathrm{u}$ \\
\hline $\mathrm{v}$ & 1.9 & - & $\mathrm{v}$ & - \\
\hline $\mathrm{w}$ & 1.2 & - & $\mathrm{w}$ & - \\
\hline $\mathrm{x}$ & 2.0 & $\mathrm{x}$ & - & - \\
\hline $\mathrm{y}$ & 1.4 & - & - & $\mathrm{y}$ \\
\hline $\mathrm{z}$ & 1.5 & - & $\mathrm{z}$ & - \\
\hline
\end{tabular}


Vertex order colouring of the fuzzy graph is

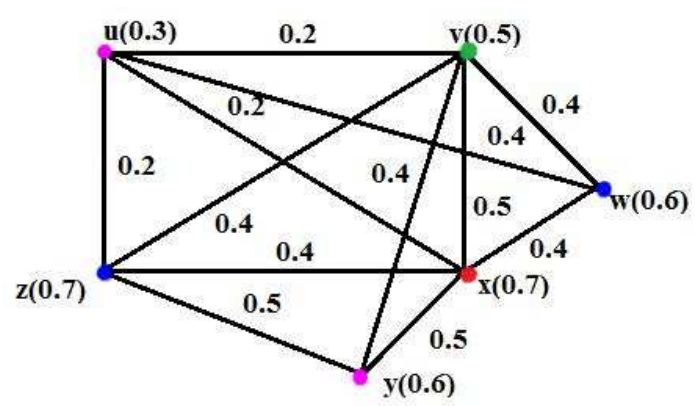

Figure 2

\begin{tabular}{|c|c|}
\hline Alpha Strong & $\mathrm{x}-1$ \\
\hline Beta Strong & $\mathrm{v}-2, \mathrm{w}-3, \mathrm{z}-3$ \\
\hline Gamma Strong & $\mathrm{u}-4, \mathrm{y}-4$ \\
\hline
\end{tabular}

Thus $\chi^{f_{o}}(G)=4$.

\section{Conclusion}

In this paper, we propose an algorithm is to determine the vertex order colouring of fuzzy graphs. Also three types of strong vertices are defined. This vertex order colouring makes easy for colouring the fuzzy graphs since colouring the graphs is widely studied area in graph theory.

\section{References}

[1] Anjaly Kishore, Sunitha M. S., Chromatic number of fuzzy graphs, Annals of Fuzzy Mathematics and Informatics, Volume x, No. x, (2013), 1-xx.

[2] Bhat. R. S., Kamath. S. S., Sureka, A Bound on Weak Domination Number Using Strong (Weak) Degree Concepts in Graphs, Journal of International Academy of Physical Sciences, Vol. 15 No. 3, (2011), 303-317.

[3] Changiz Eslahchi and B. N. Onagh, Vertex Strength Of Fuzzy Graphs, International Journal of Mathematics and Mathematical Sciences, Volume 2006, Article ID 43614, (2006), 1-9.

[4] Frank Harary, Graph Theory, Narosa /Addison Wesley, Indian Student Edition, (1988).

[5] Kamath. S. S, Bhat. R. S, Strong/Weak Neighbourhood Number of a Graph(K3 Coverings), Proc. Int. Con. on Disc. Math., Narosa Pub., New Delhi, India, (2004), 142-150. 
[6] Nagoor Gani. A., Basheer Ahamed. M., Order and Size in Fuzzy Graph, Bulletin of Pure and A lied Sciences, Vol. 22E, No. 1, (2003), 145-148.

[7] Nagoor Gani. A., Basheer Ahamed. M., Strong and Weak Domination in Fuzzy Graphs, East Asian Math. Journal, Vol. 23, No. 1, (2007), 1-8.

[8] Nagoor Gani. A., Chandrasekaran V. T., A First Look At Fuzzy Graph Theory, Allied Publishers Pvt. Ltd., (2010).

[9] Nagoor Gani. A., Radha. K, The Degree of a Vertex in some Fuzzy Graphs, International Journal of Algorithms, Computing and Mathematics, Volume 2, Number 3, (2009).

[10] Nagoor Gani. A, Radha. K, Regular Property of Fuzzy Graphs, Bulletin of Pure and A lied Sciences, Vol. 27E, No. 2, (2008), 415-423.

[11] Nivethana. V, Parvathi. A, Fuzzy Total Coloring and Chromatic Number of a Complete Fuzzy Graph, International Journal of Emerging Trends in Engineering and Development Issue 3, Vol. 6, (2013).

[12] Rosenfeld. A., Fuzzy Graphs, In:L. A. Zadeh, K. S. Fu, M. Shimura, Eds., Fuzzy Sets and Their A lications, Academic Press, (1975), 77-95.

[13] Sandeep Narayan. K. R. and Sunitha. M. S, Connectivity in a Fuzzy Graph and its Complement, Gen. Math. Notes, Vol. 9, No. 1, (2012), 38-43.

[14] Sunil Mathew, Sunitha M. S., Node Connectivity and Arc Connectivity of a Fuzzy Graph, Information Sciences, Vol. 180, issue 4, (2010), 519-531.

[15] Yeh. R. T. and Banh. S. Y., Fuzzy relations, fuzzy graphs and their a lications to clustering analysis, In Fuzzy sets and their A lications to Cognitive and Decision Process. L. A. Zadeh, K. S. Fu, M. Shimura Eds: Academic Press, New York, (1975), 125-149.

[16] Zadeh, L. A., Fuzzy Sets, Information and Control, Vol. 8, (1965), 338-353. 